\title{
Dynamic Modelling and Analysis of the Microsegment Gear
}

\author{
Yangshou Xiong, Kang Huang, Tao Wang, Qi Chen, and Rui Xu \\ School of Machinery and Automobile Engineering, Hefei University of Technology, Anhui 230009, China \\ Correspondence should be addressed to Yangshou Xiong; xiongys@mail.hfut.edu.cn
}

Received 18 June 2015; Revised 26 August 2015; Accepted 3 September 2015

Academic Editor: Radoslaw Zimroz

Copyright (C) 2016 Yangshou Xiong et al. This is an open access article distributed under the Creative Commons Attribution License, which permits unrestricted use, distribution, and reproduction in any medium, provided the original work is properly cited.

\begin{abstract}
The development of technology requires higher load capacity, rotating speed, power-weight ratio, lower vibration, and noise with respect to the gear transmission. The new type microsegment gear's tooth profile curve is composed of many microsegments. Previous researches indicate that the microsegment gear has a good static performance, while the dynamic behavior of the microsegment gear has never been investigated. This paper will focus on the dynamic performance of the gear. The profile deviation between microsegment gear and involute gear is regarded as a displacement excitation in the proposed dynamic model. The numerical analysis for three cases is conducted and the results shows that, in low-speed and heavy-load, medium-speed and medium-load conditions, microsegment gear and involute gear both exhibit a good performance, while, in high-speed and heavyload condition, microsegment gear has a better performance than that of involute gear. The influence of backlash on the dynamic performance is also studied. It is found that the variation of backlash does not change the type of motion, but the vibration amplitude and the stability of the motion are much affected. The main idea in this paper is supposed to provide a novel method for the precision grinding of the microsegment gear.
\end{abstract}

\section{Introduction}

The gear is the indispensable component of all kinds of industrial machinery, automobile, ship, locomotive, airplane, and other machines. The development of the gear has a long history since the early wooden gears applied in southpointing cart in the three Kingdoms age of china and the water device of ancient Egypt.

Involute tooth profile was first proposed by Philippe De La Hire in 1694. From then on, gear transmission has entered the new era. Its theory systems on design $[1,2]$, manufacture $[3,4]$, measurement $[5,6]$, standard [7], lubrication [8-11], and dynamics [12-17] of involute gear have been established gradually. As such, involute gear driving has developed to the most important transmission model.

Despite of taking the countermeasures such as tooth profile modification and precision grinding in many special working situation, it is still very difficult for the involute gear driving to perfectly satisfy the requirements on load capacity, rotating speed, power-weight ratio, vibration, and noise of modern industry. Involute gear thus gradually exposes some natural inadequacies such as the relative sliding between the meshing teeth (except the pitch point), and the farther the distance between mesh point and pitch point is, the greater the relative sliding velocity will be. The small curvature radius limits the bearing capacity, due to the convex-convex surfaces contact which occurred in external gear transmission.

Hence, many scholars have tried to explore and discover a kind of desirable profile to satisfy the requirements on efficiency, strength, lifespan, vibration, and noise. Actually, the researches on tooth profile have never been stopped.

In 1598, Galileo Galilei first named and mathematically defined the cycloid generating by a point on a circle rolling along a straight line or along another circle of a cycloid. Danish astronomer Olaf Roemer first proposed using cycloid as the tooth profile in 1674. Cycloidal gear has many advantages, such as the concave and convex contacting form.

Vickers-Bostock-Bramley presented a concave-convex contact gear whose profile curve actually is cycloid. The W$\mathrm{N}$ gears patented by Wildhaber (1926) and independently reinvented by Novikov (1956) are of circular profile in the transverse plane, those of the pinion being convex and those of the mating wheel concave.

In the past 30 years, Komori $[18,19]$ proposed a new type of high strength gear profile named logic gear. The logic gear profile is composed of many microsegment involutes. It has 
higher durability and strength in comparison with involute gear.

Inspired by the design strategies of logic gear, Han et al. proposed a new idea of constructing a tooth profile by discrete method. The microsegment tooth profile was first developed in 1997 [20]. The author carried out the theories and experiment researches of microsegment in his PHD dissertation. The basic characteristics such as strength problem were researched, the effect of the primary parameters on tooth profile shape was discussed, the hob cutter was designed and the strength test was conducted [21].

The temperature rise comparison test of microsegment gear and involute gear which was the first bench test for microsegment gear was conducted. The test results show that, under the same working condition, the temperature increment of microsegment gear is smaller than that of involute gear [22].

The formula to calculate transmission efficiency of microsegment gear was deduced, and the result show that the transmission efficiency of microsegment gears is higher than that of the involute gears in the same condition and the calculation result indicated that microsegment gears' transmission efficiency is little effected by gear parameters [23].

In a manner of speaking, microsegment gears have a good static performance. Nevertheless, this new gear has not been widely applied due to the structural imperfection of its theoretical system. Numerous works need to be carried out urgently such as the tooth shape detection method, precision manufacturing, and dynamic performance research.

There are many literatures which explored the dynamic performance of gear transmission, but a few of them associated with the noninvolute gear. Blagojević et al. developed a dynamic model of a single-stage cycloid drive and considered the dynamic behavior of cycloid planetary gear trains [24]. As to the dynamic analysis of microsegment gear, it is still a blank.

Consequently, this paper will focus on the dynamic performance of microsegment gear transmission, including establishing the dynamic model for microsegment gear and investigating the nonlinear dynamics in different working conditions. The time-varying mesh stiffness and backlash have also been taken into account. We have great expectations to lay a foundation for microsegment gear dynamic theoretical system.

\section{Review of Microsegment Gear}

The tooth profile curve of the gear's generating cutting gears is enveloped by lots of lines; thus the truly tooth profile is composed of a large number of microsegments. Inspired by this, the microsegment gear tooth profile was proposed. The basic rack should be constructed firstly, and then the tooth profile can be obtained using the principle of generating cutting; see Figure 1.

In Figure 1, the rays $O_{1} N_{1}$ and $O_{1} n_{0}$ intersect to each other at point $O_{1}$ with angle $\alpha_{0}$. The pitch line $t t$ is perpendicular

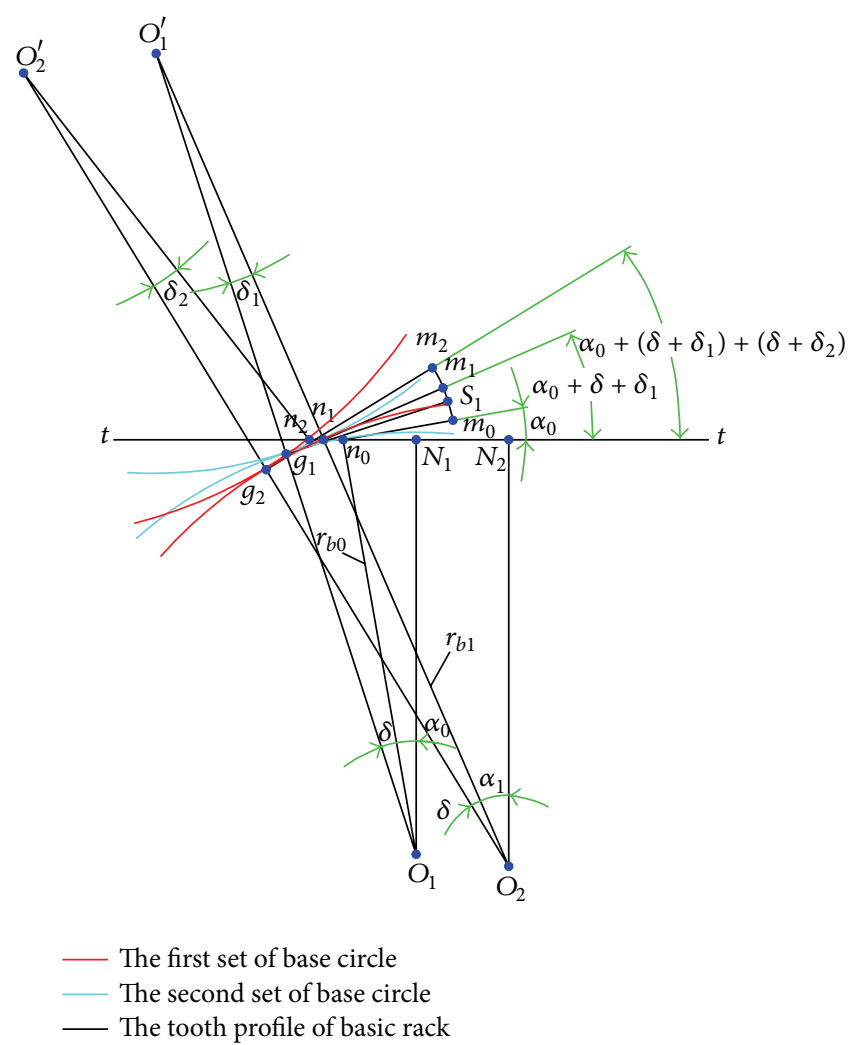

FIgURE 1: The principle of microsegment gear tooth profile.

to $O N_{1}$ and intersects with two rays at $N_{1}$ and $n_{0}$ and the distance between point $O_{1}$ and point $n_{0}$ is $r_{b 0}$. The length of line $O_{1} O_{1}^{\prime}$ equals $2 r_{b 0}$ and the angle $\delta$ is selected in advance. Two base circles with radius at $r_{b 0}$ are tangent to each other and intersect with pitch line $t t$ at point $n_{0}$ and $n_{1}$, respectively. The line $g_{1} S_{1}$ is supposed to be the line of action (LOA). The arc $S_{1} m_{0}$ and $S_{1} m_{1}$ are the microsegments generated by line $g_{1} S_{1}$ rolling along each base circle for an arc length of $g_{1} n_{0}$ and $g_{1} n_{1}$ according to the involute forming principle. Thus, the curvature centers of two points $m_{0}$ and $m_{1}$ (also named null-point as the comprehensive curvature of these kinds of points is zero) locate at points $n_{0}$ and $n_{1}$, and the radii of curvature should be $\rho_{m 0}=m_{0} n_{0}, \rho_{m 1}=$ $m_{1} n_{1}$. The constructing process of microsegment gear tooth profile is a recursive process with a proper sequence. For every microsegment, the pressure angle has an increment compared to the last one. The curvature centers of nullpoints all locate on pitch line, and the adjacent curves are smooth and continuous at null-points. Then line $O_{1}^{\prime} O_{2}$ is made through point $O_{1}^{\prime}$ with angle $\delta$ from line $O_{1} O_{1}^{\prime}$ which intersects pitch line at point $n_{1}$. The microsegment $m_{1} m_{2}$ will be obtained by repeating above process of $m_{0} m_{1}$ when $\mathrm{O}_{2} n_{1}=r_{b 1}$. The tooth profile curve will be formed by repeating the constructing process indefinitely and the final profile will obtained according to the positions of addendum and dedendum circle. For example, the final tooth profile curve contains 789 microsegments for a spur microsegment gear with the parameters of the tooth number $z=30$ and module $m=2$. 


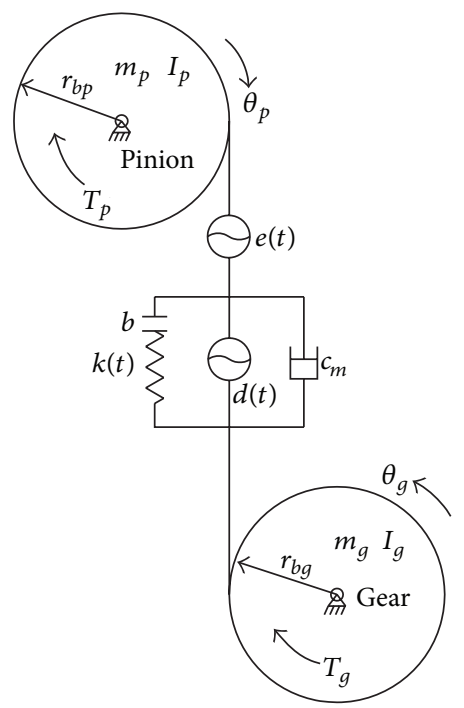

FIGURE 2: The nonlinear dynamic model of the two gears system.

\section{Dynamic Model for Microsegment Gear}

According to the above constructing principle, it is easy to find out that the base circle of microsegment tooth profile disciplinary changes from one microsegment to the next, while the involute tooth profile has an invariable base circle. The varying base circle means varying line of action and direction of meshing force. The meshing process of microsegment gear thus becomes extremely complex.

Many researches have devoted to the dynamic modelling of the involute gear system [25]. The incomplete model with two gear wheels (SDOF or MDOF) has been widely approved and applied [12-15, 26-28]. Thus, we propose a dynamic model of microsegment gear based on SDOF model, and the tooth profile deviation between microsegment gear and involute gear is regarded as a displacement excitation.

3.1. Mathematical Representation of Dynamic Model for Microsegment Gear Pair. The mechanical model is shown in Figure 2. According to the SDOF torsional model, each gear body is combined with its supporting shaft into a single rigid body given to a great bearing stiffness. As usual, the influence of the friction and the fluctuation of the torques are ignored. Different from the involute gear torsional model, presented model regards the tooth profile deviation as displacement excitation.

Then employing the composite coordinate

$$
x(t)=r_{b p} \theta_{p}-r_{b g} \theta_{g}-d(t)-e(t)
$$

results in the elimination of the rigid-body rotation of the original model and yields a single equation of motion in the form

$$
m_{e} \ddot{x}(t)+c_{m} x \dot{(t)}+k(t) h(x(t))=F_{m}-F(t)
$$

with

$$
\begin{aligned}
m_{e} & =\frac{I_{g} I_{p}}{\left(I_{g} r_{b p}^{2}+I_{p} r_{b g}^{2}\right)}, \\
F_{m} & =\frac{T_{p}}{r_{b p}}=\frac{T_{g}}{r_{b g}}, \\
F(t) & =m_{e}(e \ddot{(t)}+d \ddot{(t} t),
\end{aligned}
$$

where $I_{p}, I_{g}$ are polar mass moments of inertia. $m_{p}, m_{g}$ are mass of each body. $T_{p}, T_{g}$ are drive torque and load torque. $r_{b p}$ and $r_{b g}$ are base radii of involute gears which have the same teeth number and module with microsegment gears. $k(t)$ is varying mesh stiffness of the gear set. $e(t)$ is static transmission error. $d(t)$ is the tooth profile deviation of microsegment gear comparing with involute gear. The mesh stiffness, static transmission error, and tooth profile deviation can approximately be considered as time-periodic functions. $c_{m}$ is damping between meshing teeth which is proportional to the equivalent mesh stiffness $k_{m}$ and can be evaluated approximately using the following equation:

$$
c_{m}=2 \zeta \sqrt{\frac{k_{m}}{1 / m_{p}+1 / m_{g}}}
$$

with the damping ratio $\zeta=0.03 \sim 0.17$.

$h(x)$ is backlash function, which is usually calculated by

$$
h(x(t))= \begin{cases}x(t)-b, & x(t)>b \\ 0, & |x(t)|<b \\ x(t)+b, & x(t)<-b,\end{cases}
$$

where $b$ represents the backlash of gear pair and its value is about 100 400 $\mu \mathrm{m}$ according to DIN 3967-1978.

3.2. Two Important Parameters in Dynamic Model $(k(t), d(t))$. The main difference between proposed dynamic model and the traditional SDOF is embodied in two important parameters $k(t)$ and $d(t)$.

3.2.1. Time-Varying Mesh Stiffness $k(t)$. The gear stiffness and its changing characteristics are always the crucial basis of gear dynamics in meshing process. It can be divided into tooth stiffness, the tooth mesh stiffness, and comprehensive mesh stiffness. The comprehensive mesh stiffness considers many factors, such as the meshing position and the contact ratio. In normal contact ratio gear transmission, the situations of one pair of tooth in contact and two pairs of tooth in contact occur alternately in one mesh period.

As we all know, the tooth stiffness can be expressed as

$$
\begin{aligned}
& k_{p i}=\frac{F}{\delta_{p i}}, \\
& k_{g i}=\frac{F}{\delta_{g i}},
\end{aligned}
$$


TABLE 1: Common design parameters of the spur gear pairs.

\begin{tabular}{lc}
\hline Parameter & Pinion/gear \\
Number of teeth & 30 \\
Transverse module $(\mathrm{mm})$ & 2 \\
Width $(\mathrm{mm})$ & 20 \\
Mass $(\mathrm{kg})$ & 0.385 \\
Moments of inertia $\left(\mathrm{kg} \cdot \mathrm{m}^{2}\right)$ & $1.92 e-4$ \\
Damping ratio $\zeta$ & 0.12 \\
Elasticity modulus $(\mathrm{Gpa})$ & 205 \\
Poisson ratio & 0.3 \\
\hline
\end{tabular}

where $i$ is the maximum number of meshing tooth of each gear at the same time, $F$ is applied load, and $\delta$ is deflections of the tooth in the direction of applied load.

The tooth mesh stiffness can be expressed as

$$
k_{i}=\frac{k_{p i} \cdot k_{g i}}{k_{p i}+k_{g i}} .
$$

The comprehensive mesh stiffness is

$$
k=\sum_{j=1}^{i} k_{j} .
$$

From the available literature, different methods and empirical equations are used to calculate the comprehensive mesh stiffness. These methods are often based on the classical theory of elasticity and numerical approaches. However, it is considered to be unsuitable to use these methods on the dynamic research of microsegment gear due to the special profile.

Therefore, the 3D finite element method is utilized to calculate the tooth stiffness for both microsegment gear and involute gear to make a comparison. With the design parameters of microsegment gears listed in Table 1, the 3D finite element model for a pair of tooth of microsegment gear is shown in Figure 3.

The 3D finite element model contains about 43870 elements. Four end faces and one cylindrical face are fixed, and the other cylindrical face is defined as the loading surface. Since the meshing process is nonlinear, the contact surfaces are defined as nonlinear contacting to get a more realistic result.

The standard involute gears used to make a comparison have the same number of teeth and transverse module with the analyzed microsegment gear pair. The deformation and stiffness of these two kinds of profile are shown in Figure 4. It is easy to find out that the mesh stiffness of microsegment gear is better than that of involute gear's in the same parameters.

In the process of meshing, the mesh stiffness presented the obvious periodicity; then it can be expressed in the form of Fourier series expansion as follows:

$$
k(t)=k_{m}+\sum_{j=1}^{\infty}\left[k_{a j} \cos \left(j w_{e} t\right)+k_{b j} \sin \left(j w_{e} t\right)\right],
$$

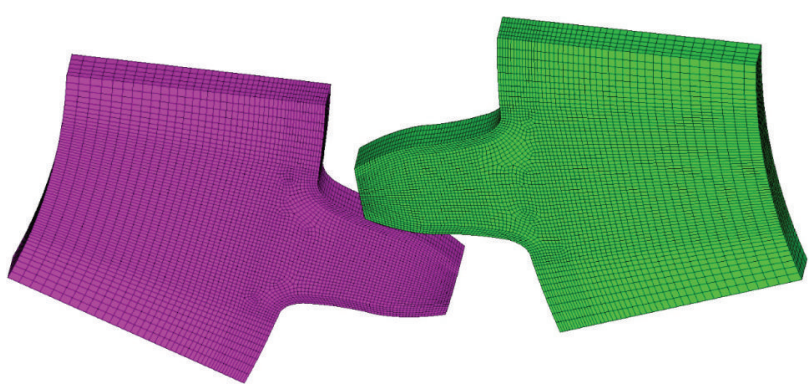

FIgURE 3: The 3D finite element model of microsegment gear.

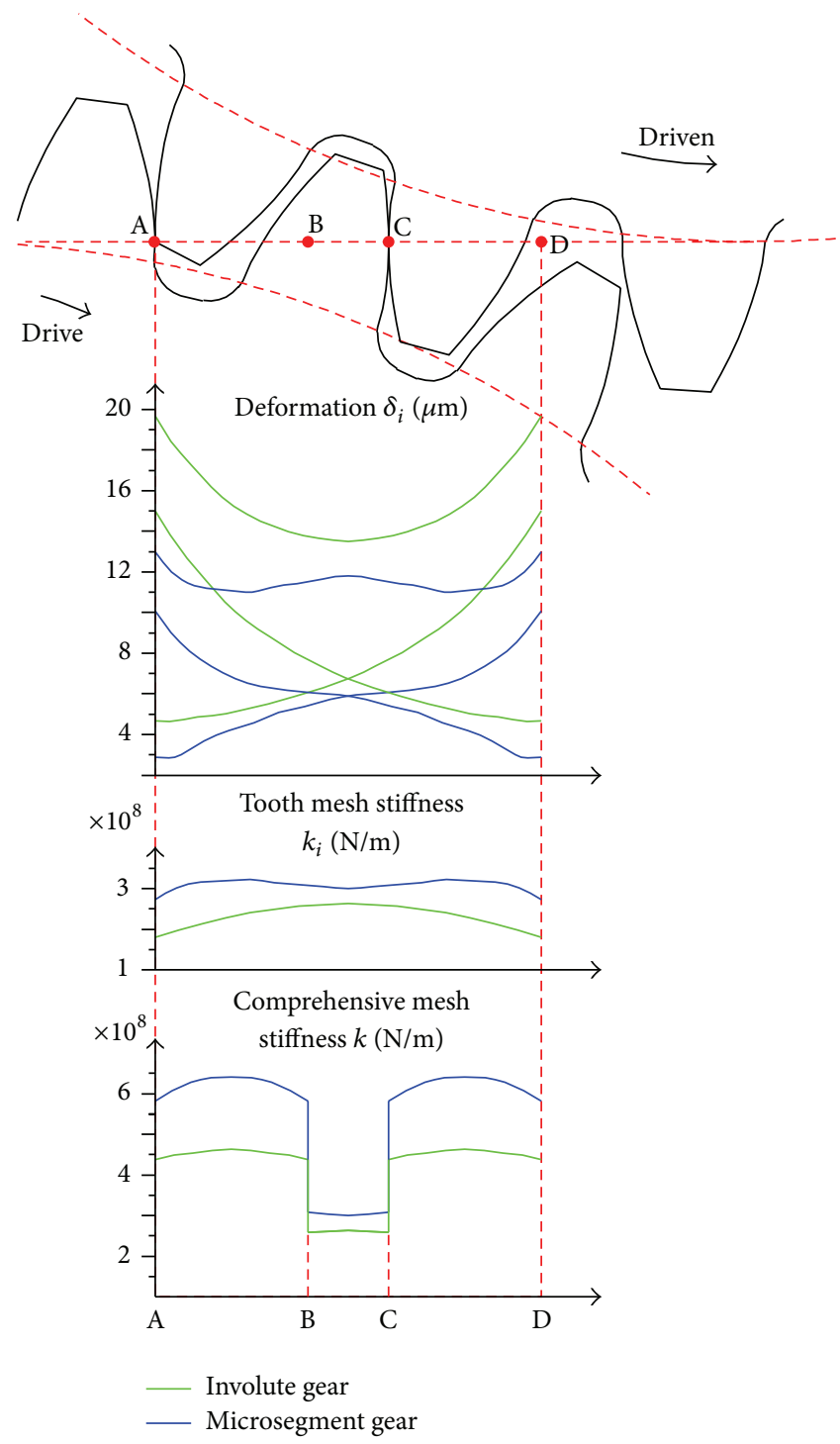

FIGURE 4: The deformation and stiffness of microsegment gear and involute gear.

where $k_{m}$ is average mesh stiffness. $k_{a j}$ and $k_{b j}$ are amplitudes of the jth order harmonic. $w_{e}$ is mesh frequency. It can work out that the first six-order Fourier series is sufficient to fit the actual time-varying mesh stiffness and each harmonic parameter of the meshing stiffness is listed in Table 2. 


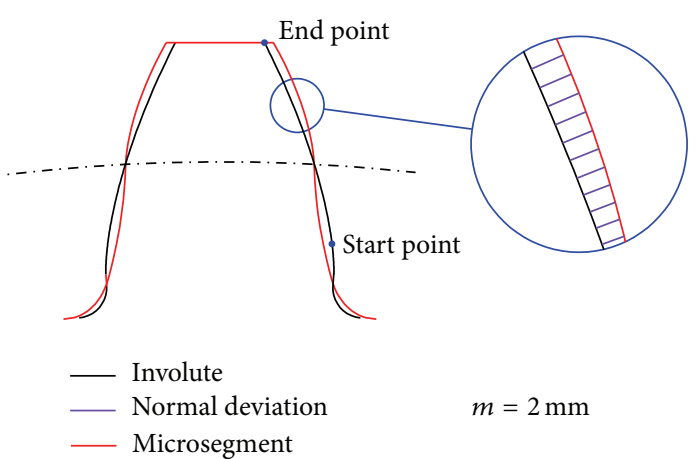

FIGURE 5: The geometric models for both microsegment gear and standard involute gear.

TABLE 2: Each harmonic parameter of meshing stiffness $\left(10^{8} \mathrm{~N} / \mathrm{m}\right)$.

\begin{tabular}{ccccc}
\hline$j$ & \multicolumn{2}{c}{ Microsegment gear } & \multicolumn{2}{c}{ Involute gear } \\
& $k_{a j}$ & $k_{b j}$ & $k_{a j}$ & $k_{b j}$ \\
\hline 1 & 0.8008 & 1.176 & -0.2568 & 0.9511 \\
2 & 0.3731 & -0.9478 & 0.4661 & 0.2714 \\
3 & -0.4555 & 0.1032 & 0.1328 & -0.1314 \\
4 & 0.03526 & 0.03286 & 0.05432 & 0.09573 \\
5 & -0.02101 & 0.1369 & 0.223 & -0.05751 \\
6 & 0.2532 & -0.121 & 0.001915 & -0.1706 \\
\hline$k_{m}$ & \multicolumn{3}{c}{3.472} & \multicolumn{3}{c}{}
\end{tabular}

3.2.2. Profile Deviation $d(t)$. In this subsection, the normal deviation of microsegment gear comparison with the standard involute profile will be analyzed. In Figure 5, the geometric models of the single tooth for both microsegment gear and standard involute gear are established. And it is clear that these two profiles are obviously different.

To research the influence of different profiles to the dynamic characteristics of the gear system, the magnitude of deviation should be accurately expressed. The mesh cycle of one tooth is moving from the start point to the end point. The angle of rotation of one mesh cycle is divided into many small pieces, and each angle corresponds to a normal deviation, as show in Figure 5. Due to the cooperative working of two gears, the displacement excitation $d(t)$ should be the resultant of normal deviation of the gear and pinion body, as shown in Figure 6.

Identical to the time-varying mesh stiffness, the synthetical normal deviation also presents an obvious periodicity. And it can also be expressed as first-order Fourier series expansion $(\mathrm{mm})$ :

$$
d(t)=-0.0015+0.01 \cos \left(w_{e} t\right)+0.0157 \sin \left(w_{e} t\right) .
$$

3.3. Nondimensionalization of the Dynamic Differential Equation. As usual, the vibration differential equation of the gear system is processed to be dimensionless to assure that the equation does not dependent on the specific physical dimension. Then define a dimensionless time $\tau=t \cdot \omega_{n}$ and

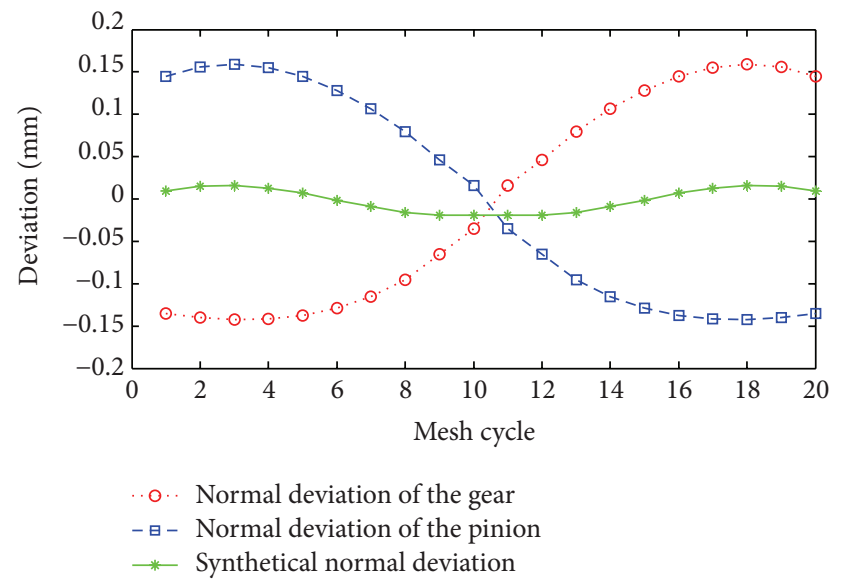

FIgURE 6: The normal profile deviation of microsegment gear compared with standard involute gear.

dimensionless displacements $l$. Giving $\bar{b}=b / l, \bar{e}(\tau)=e(t) / l$, $\bar{d}(\tau)=d(t) / l, \bar{x}(\tau)=x(t) / l$, and it can deduced that

$$
\begin{aligned}
& x \dot{(t)}=l \omega_{n} \bar{x} \dot{(\tau),} \\
& \dot{d(t)}=l \omega_{n} \bar{d} \dot{(\tau)}, \\
& x \ddot{(}(t)=l \omega_{n}{ }^{2} \bar{x} \ddot{(\tau),} \\
& \left.e \ddot{e}(t)=l \omega_{n}{ }^{2} \bar{e} \ddot{\tau}\right), \\
& d \ddot{d}(t)=l \omega_{n}{ }^{2} \bar{d} \ddot{(\tau)} .
\end{aligned}
$$

Here, the static transmission error $e(t)$ is given $e(t)=$ $e_{m} \cos \left(\omega_{e} t\right)$.

Then the original equation of motion (2) can eventually be put in the normalized form

$$
\begin{aligned}
& \bar{x}(\tau)+\xi \bar{x} \dot{(\tau)} \\
& \quad+\left(1+\sum_{j=1}^{6}\left(\gamma_{a j} \cos (\Omega \tau)+\gamma_{b j} \sin (\Omega \tau)\right)\right) h(\bar{x}(\tau)) \\
& =F-(\bar{e} \ddot{(} \tau)+\bar{d} \ddot{(\tau)}),
\end{aligned}
$$


Backlash $b=100 \mu \mathrm{m}$

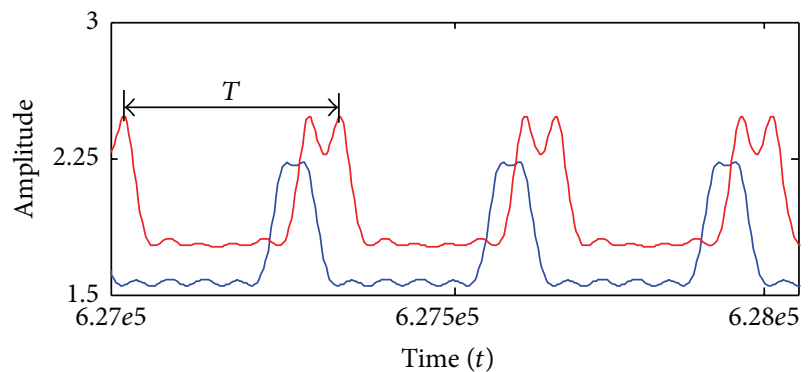

— Microsegment

_ Involute

(a) Time history chart

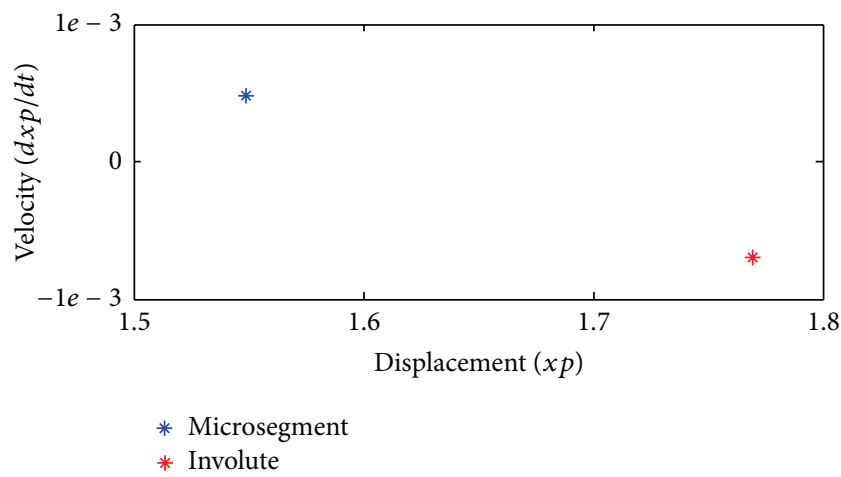

(c) Poincare perception mapping

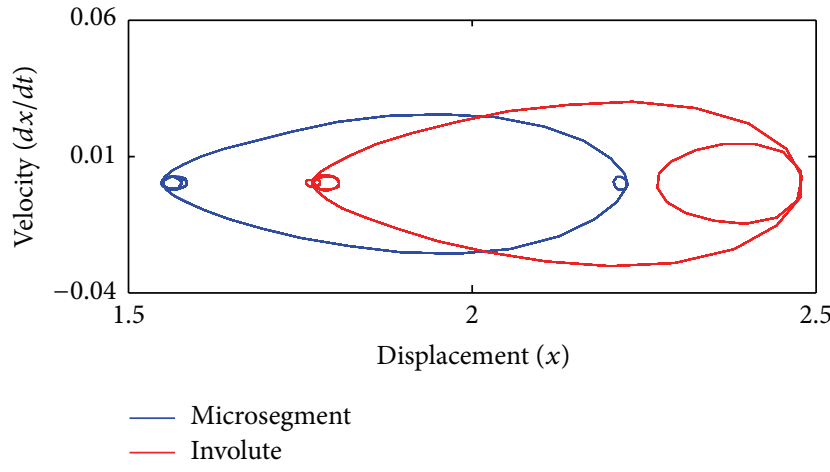

(b) Phase diagram

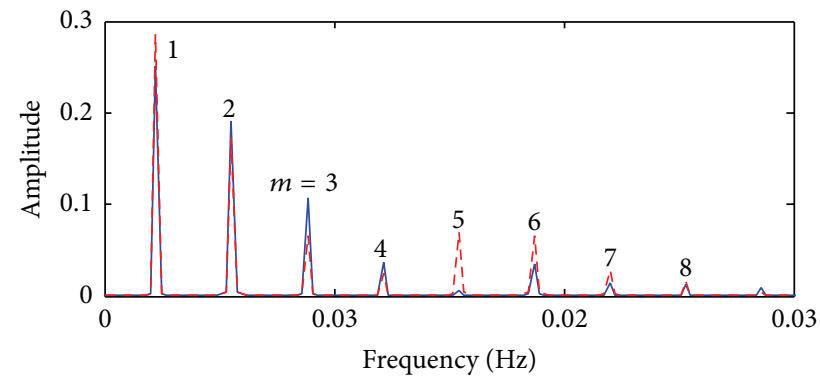

— Microsegment

- - - Involute

(d) FFT spectrum

Backlash $b=200 \mu \mathrm{m}$

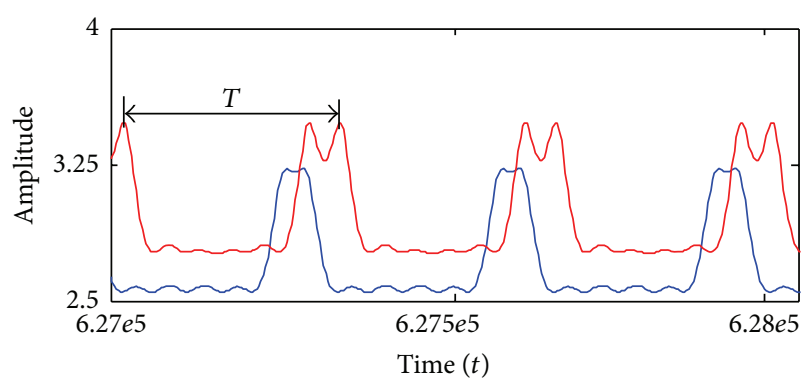

— Microsegment

— Involute

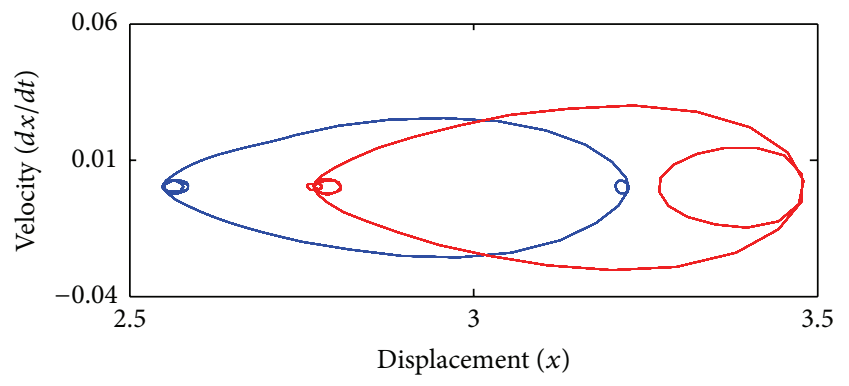

- Microsegment

_ Involute

(b) Phase diagram

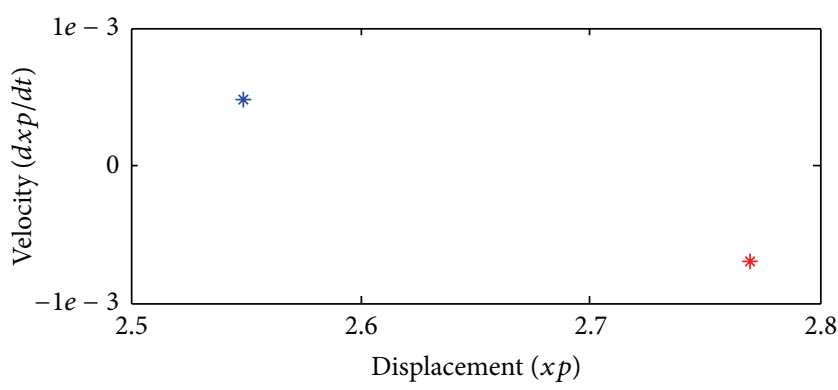

* Microsegment

* Involute

(c) Poincare perception mapping

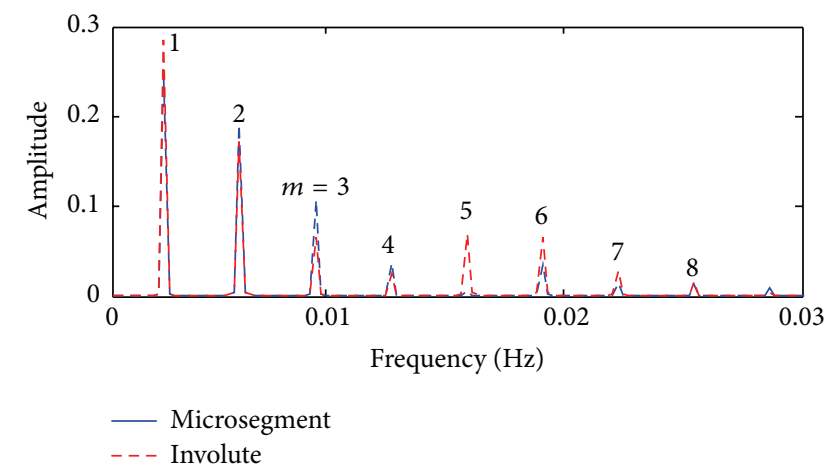

(d) FFT spectrum

Figure 7: Continued. 


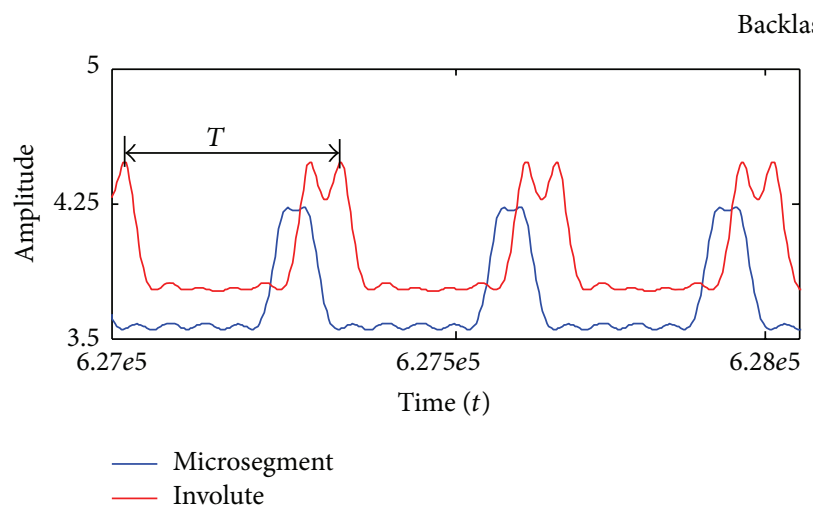

(a) Time history chart

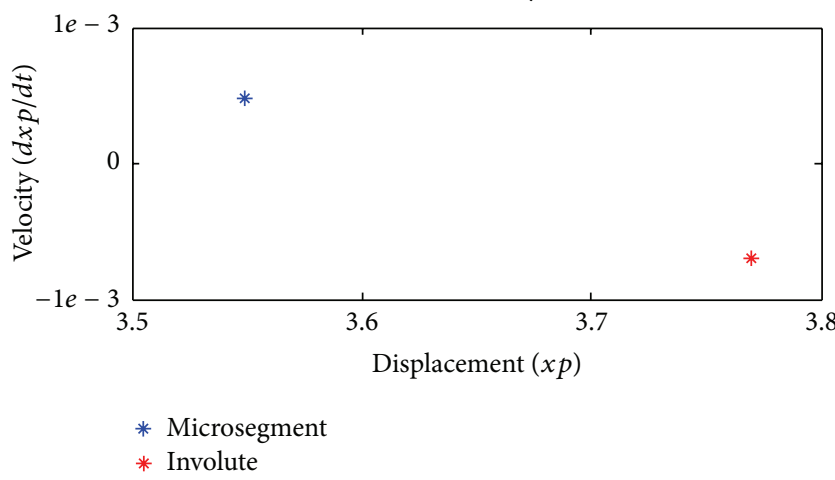

(c) Poincare perception mapping

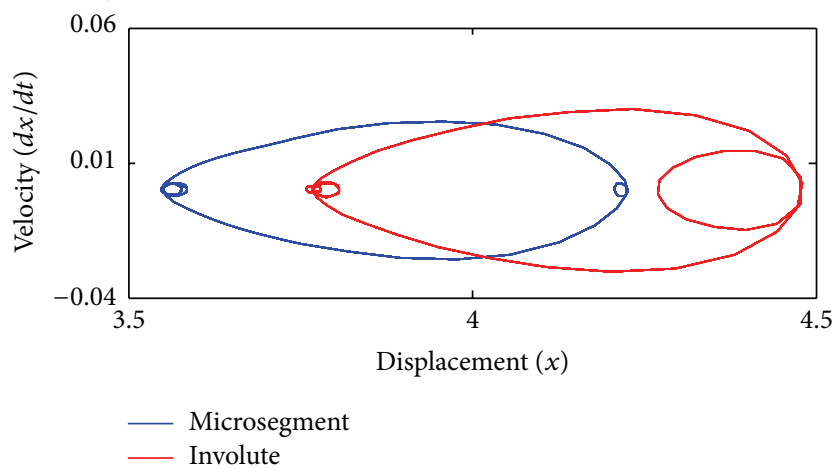

(b) Phase diagram

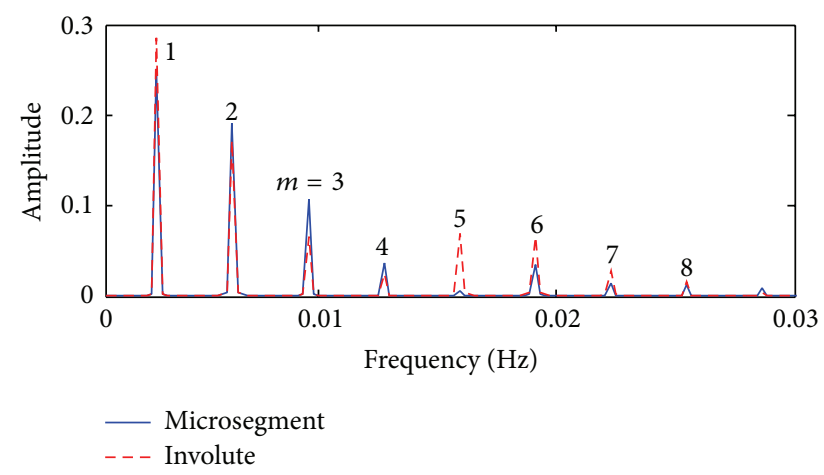

(d) FFT spectrum

Figure 7: Dynamic performance comparison at $\Omega=0.02, T=1000 \mathrm{~N} \cdot \mathrm{m}$.

$$
h(\bar{x}(\tau))= \begin{cases}\bar{x}(\tau)-\bar{b}, & \bar{x}(\tau)>\bar{b} \\ 0, & |x(\tau)| \leq \bar{b} \\ \bar{x}(\tau)+\bar{b}, & \bar{x}(\tau)<\bar{b}\end{cases}
$$

where $\xi$ is dimensionless damping ratio of tooth mesh $(\xi=$ $\left.c_{m} / m_{e} \omega_{n}\right), \gamma_{a j}, \gamma_{b j}$ are the time-variant stiffness coefficients $\left(\gamma_{a j}=k_{a j} / k_{m}, \gamma_{b j}=k_{b j} / k_{m}\right), F$ is dimensionless excitation $\left(F=F_{m} / m_{e} l \omega_{n}^{2}\right)$, and $\Omega$ is dimensionless mesh frequency $\left(\Omega=\omega_{e} / \omega_{n}\right)$.

\section{Numerical Results and Discussion}

To enhance the practical engineering value, three different cases of working condition are simulated to investigate the influence of backlash on the dynamic characteristics. Both microsegment gear and involute gear are investigated to make a comparison. Generally, the analysis results reflecting system behaviors from different perspective can be got by the nonlinear vibration method, such as the time process diagram, phase diagram, Poincare maps, and FFT spectrum. A stable response induced by different excitation ( $T$ as mesh period and $w_{e}$ as mesh frequency) can be classified into five categories according to the analysis results, (a) simple harmonic response, (b) nonharmonic single periodic response, (c) subharmonic response, (d) quasiperiodic response, and (e) chaotic responses.

\subsection{Simulation with Low-Speed and Heavy-Load (LSHL)}

Case $1(\Omega=0.02, T=1000 \mathrm{~N} \cdot \mathrm{m})$. This case is aimed at simulating the gears working in LSHL condition. According to Figure 7 , it is clear to find out that the microsegment gear system is under a status of nonharmonic single periodic response. The response period $T$ of time history chart equals the excitation period, the phase graph is a noncircle and nonelliptic curve, the resulting trace in Poincare map is a dot, and the discrete points locate at the frequencies of $m \omega_{e}(m$ is a positive integer, similarly hereinafter) in corresponding FFT spectrum according to Figure 7. As the magnitude of backlash increases from $100 \mu \mathrm{m}$ to $300 \mu \mathrm{m}$, the periodicity of the system changes a little except the vibration amplitude in time history chart moves up appropriately.

Compared with vibration response of microsegment gear, the involute gear's presents the same situation: a nonharmonic single periodic response. The comparison results show that the vibration amplitude of involute gear in each backlash is greater than that of microsegment gear.

\subsection{Simulation with Medium-Speed and Medium-Load (MSML)}

Case $2(\Omega=0.2, T=200 \mathrm{~N} \cdot \mathrm{m})$. The working condition in this case is a very common for most machines. The numerical calculation results also show two nonharmonic single periodic responses according to Figure 8. 

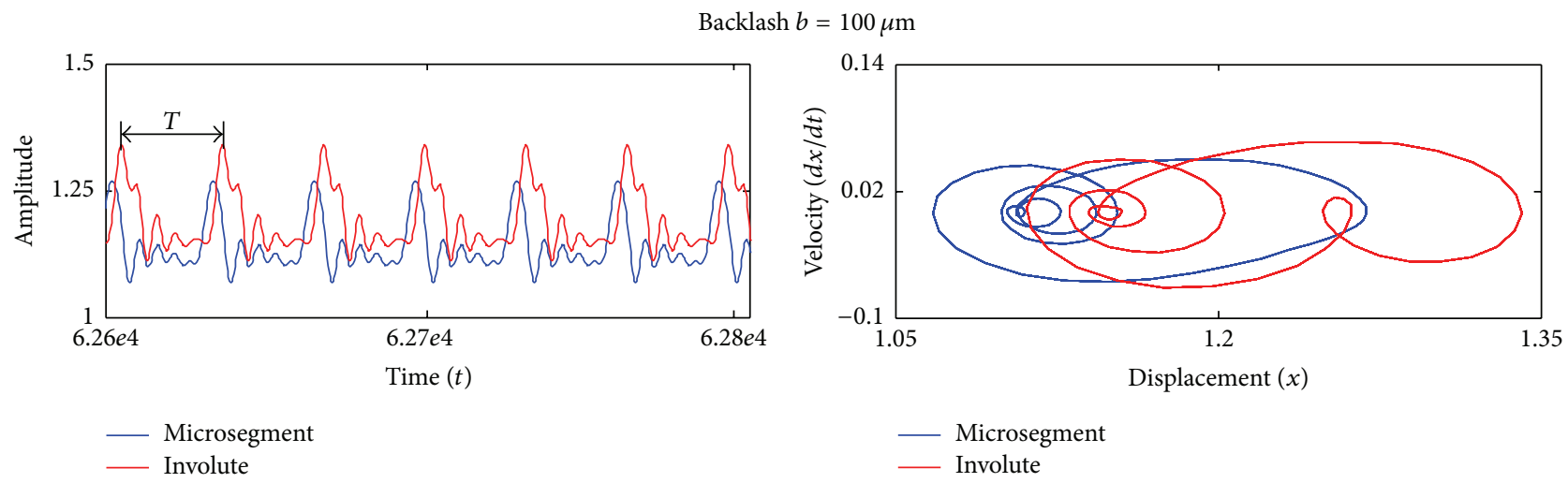

(a) Time history chart

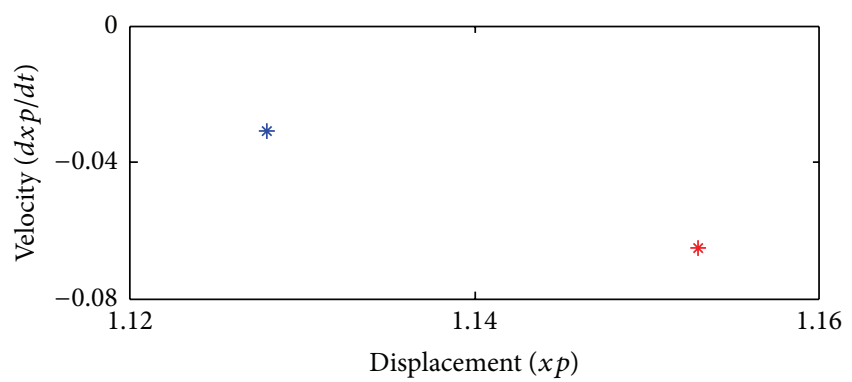

(b) Phase diagram

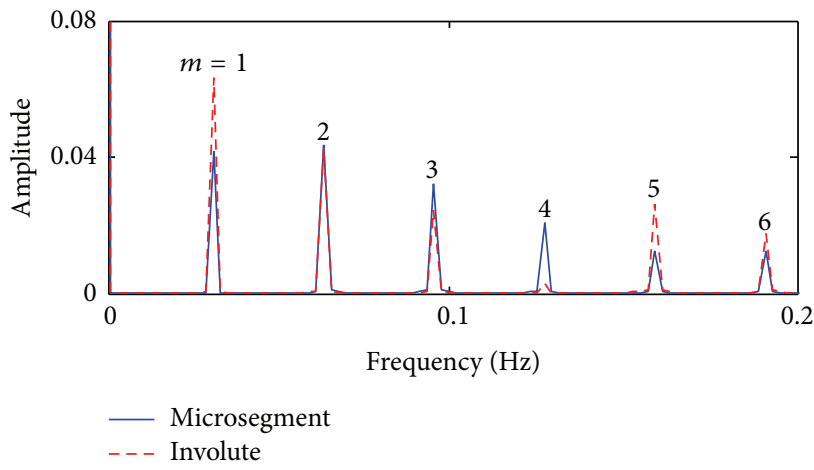

(c) Poincare perception mapping

(d) FFT spectrum

Backlash $b=200 \mu \mathrm{m}$
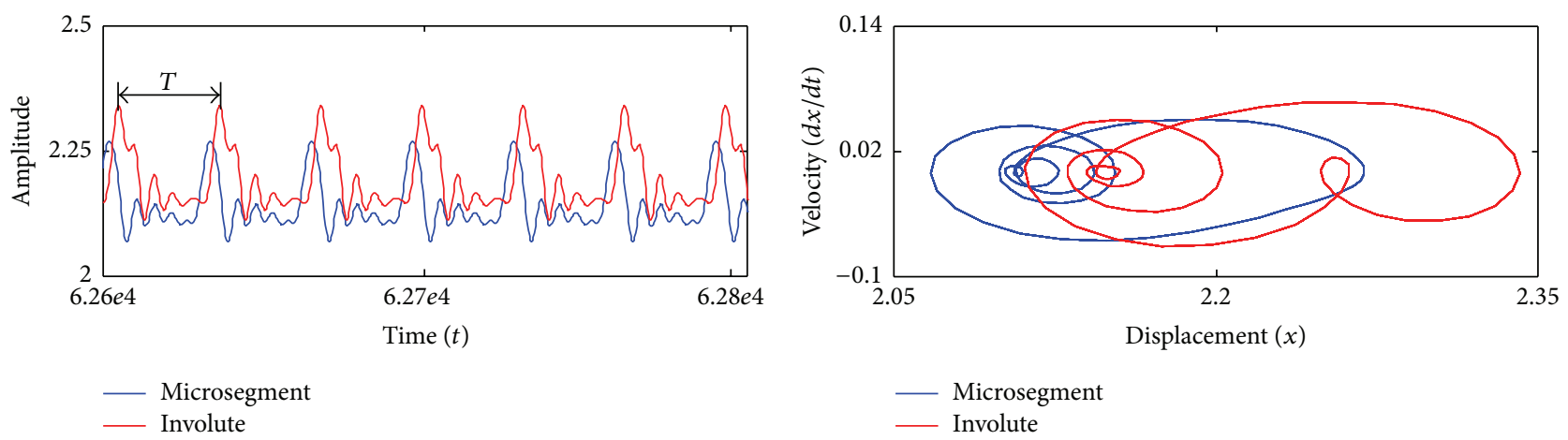

(a) Time history chart

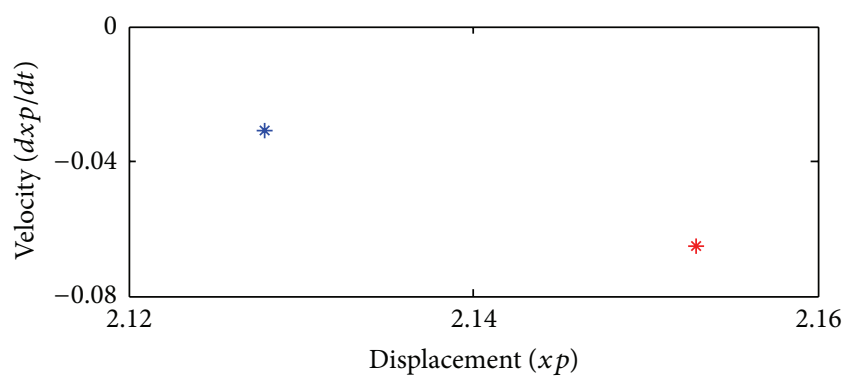

(b) Phase diagram

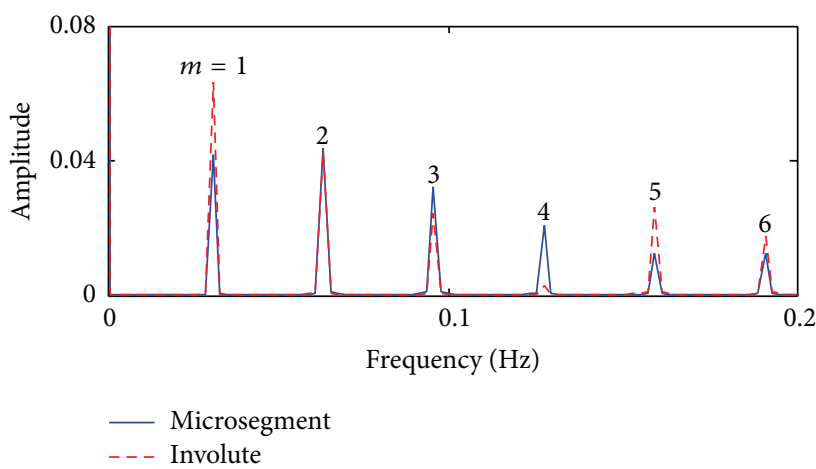

(c) Poincare perception mapping

(d) FFT spectrum

Figure 8: Continued. 

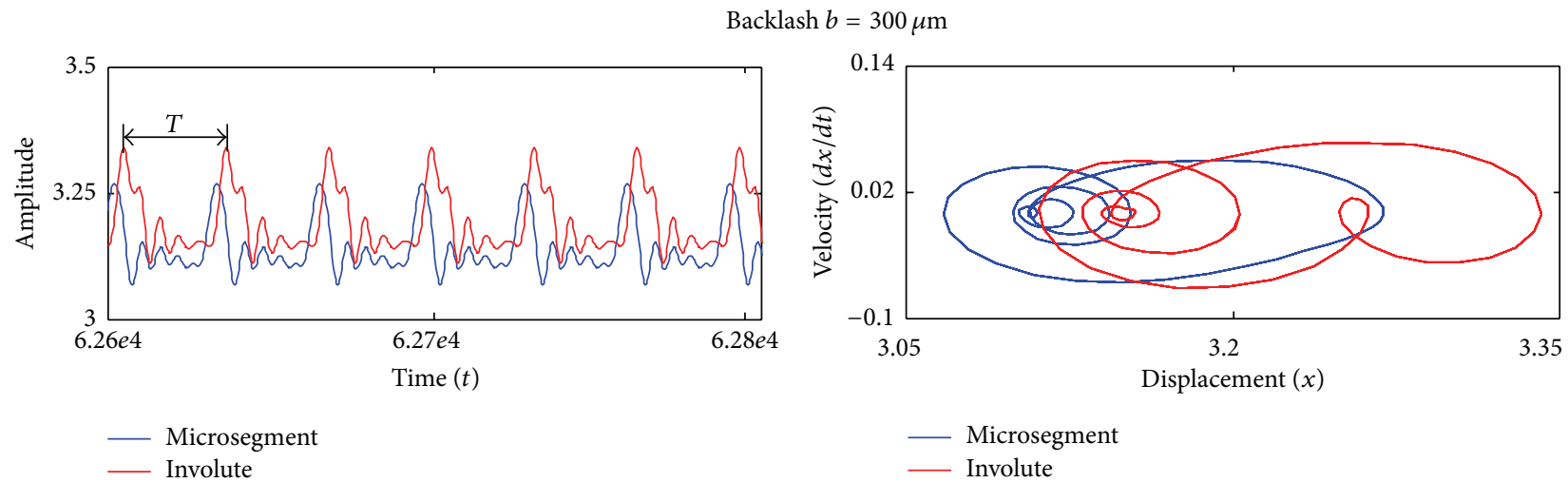

(a) Time history chart
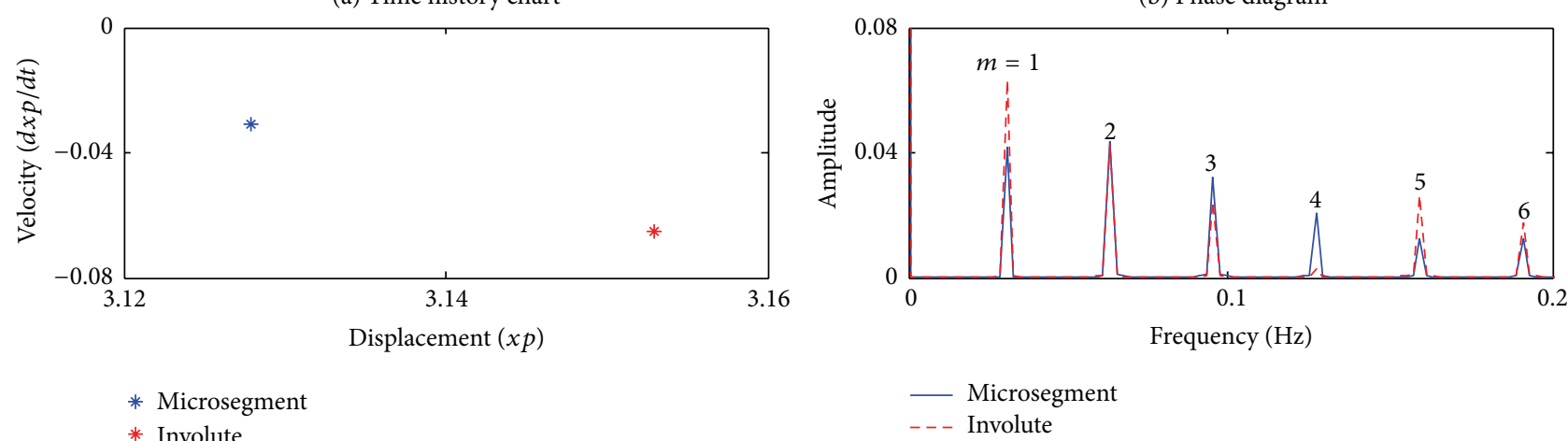

(c) Poincare perception mapping

(d) FFT spectrum

FIgURE 8: Dynamic performance comparison at $\Omega=0.2, T=200 \mathrm{~N} \cdot \mathrm{m}$.

It is hard to find obvious differences from the comparison of two profile. Both of them have a strong cyclical characteristics. The vibration range shows the same situation as Case 1 , and the dynamic performance is little affected by the backlash.

\subsection{Simulation with High-Speed and Heavy-Load (HSHL)}

Case $3(\Omega=2, T=1000 \mathrm{~N} \cdot \mathrm{m})$. This case represents the HSHL working condition. And the dynamic characteristics of microsegment gear and involute gear are revealed in Figure 9. Two simple harmonic responses are exhibited according to the figures. The period and frequency of the motion equal these of excitation. When the backlash is $100 \mu \mathrm{m}$, the motions of microsegment gear show great periodicity and stability. As the backlash increases to $200 \mu \mathrm{m}$, the motion tends to be worse according to the degree of concentration of the points in Poincare map, but the situation is getting better as the backlash further increases to $300 \mu \mathrm{m}$. In a manner of speaking, we cannot get the conclusion based on the dynamics: the dynamic performance is not always better as the backlash becomes smaller.

Compared with the microsegment gear, the involute gear working in this condition does not show a good dynamic performance according to the periodicity and stability exhibiting in the phase diagram and Poincare map.
In the manner of speaking, the modelling method here is acceptable, as the contrast analyses above show that these two type of gears perform the same responses in three cases and the SDOF model for involute gear has been widely approved.

However, the difference between involute and microsegment gears is not obvious. To give a more clear idea about the difference, the dynamic meshing loads and the Root-MeanSquare (RMS) of the vibration amplitude are employed.

The dimensionless dynamic meshing loads in formula (12) is $\left(1+\sum_{j=1}^{6}\left(\gamma_{a j} \cos (\Omega \tau)+\gamma_{b j} \sin (\Omega \tau)\right)\right) h(\bar{x}(\tau))$ which can be plotted as Figure 10 shows.

According to Figure 10, it is clear that the microsegment gears have the disadvantage of reducing the dynamic meshing loads, especially in Cases 1 and 2.

The effective value of vibration signal can be expressed in terms of RMS value which can be defined as

$$
A^{\mathrm{RMS}}=\sqrt{\frac{\sum_{i=1}^{N}\left(\text { amplitude }^{(i)}\right)^{2}}{N}},
$$

where amplitude ${ }^{(i)}$ is the $i$ th harmonic amplitude, and here $i=8$ is found sufficient. Then the RMS of amplitude can be obtained, as shown in Figure 11.

Figure 11 gives a clear view of the comparison of the $A^{\mathrm{RMS}}$. For the most of the condition, the $A^{\mathrm{RMS}}$ of microsegment gear is lower than that of involute gear. 
Backlash $b=100 \mu \mathrm{m}$
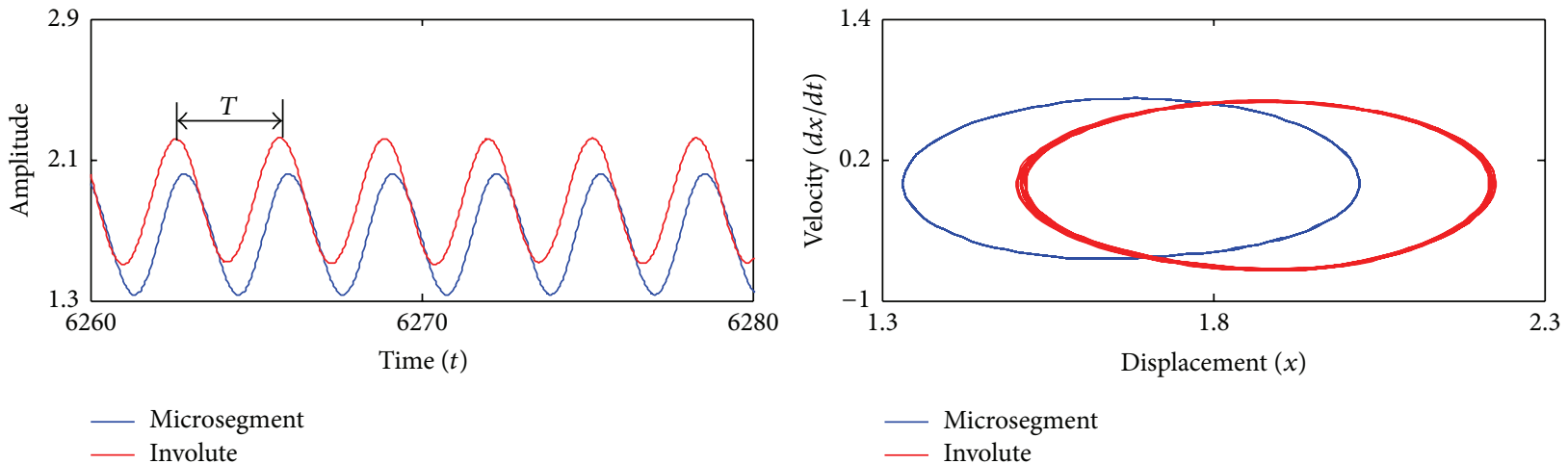

(a) Time history chart
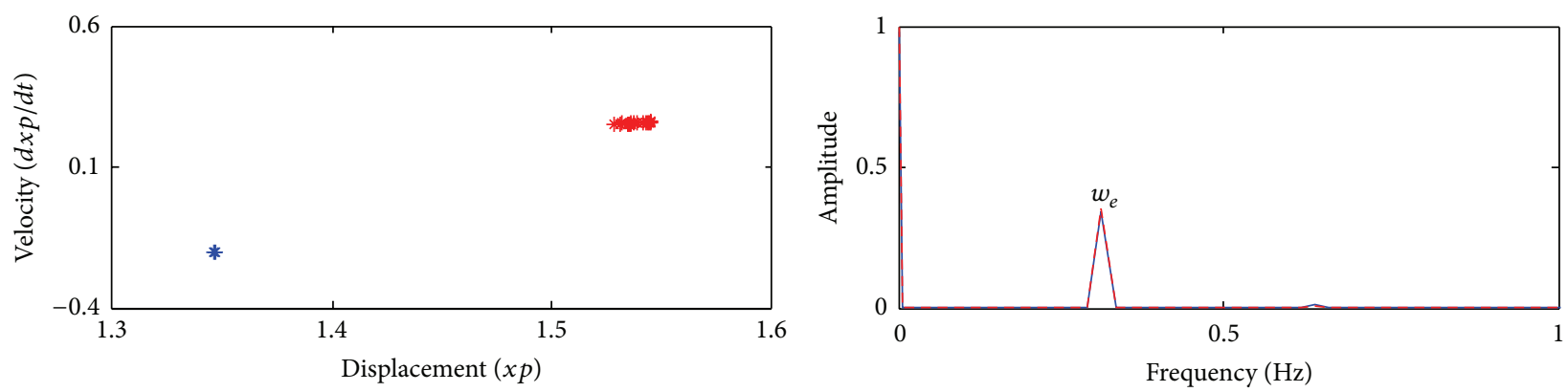

* Microsegment

* Involute

__ Microsegment

(c) Poincare perception mapping

(d) FFT spectrum

Backlash $b=200 \mu \mathrm{m}$
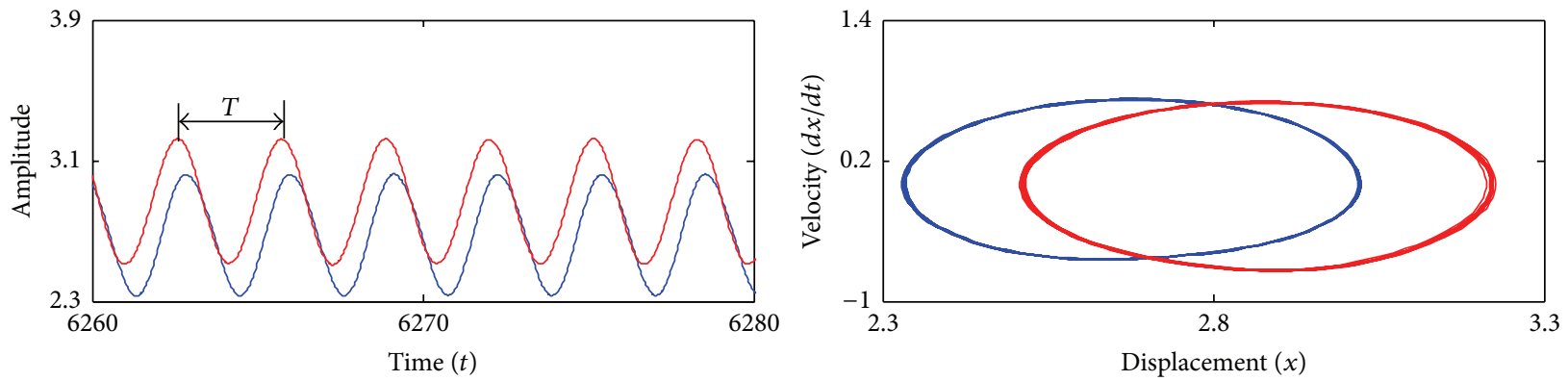

— Microsegment

_ Involute

(a) Time history chart

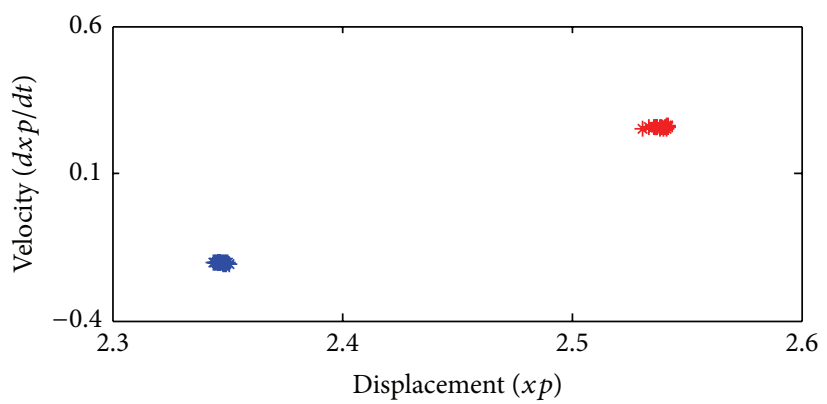

* Microsegment

* Involute

(c) Poincare perception mapping
— Microsegment

Involute

(b) Phase diagram

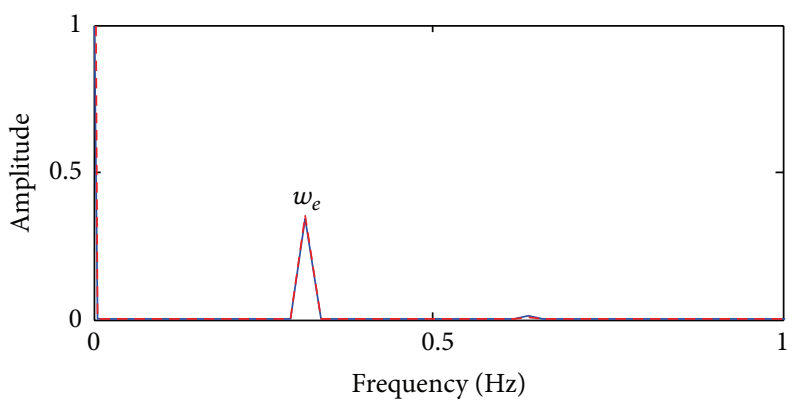

_ Microsegment

- - - Involute

(d) FFT spectrum

Figure 9: Continued. 

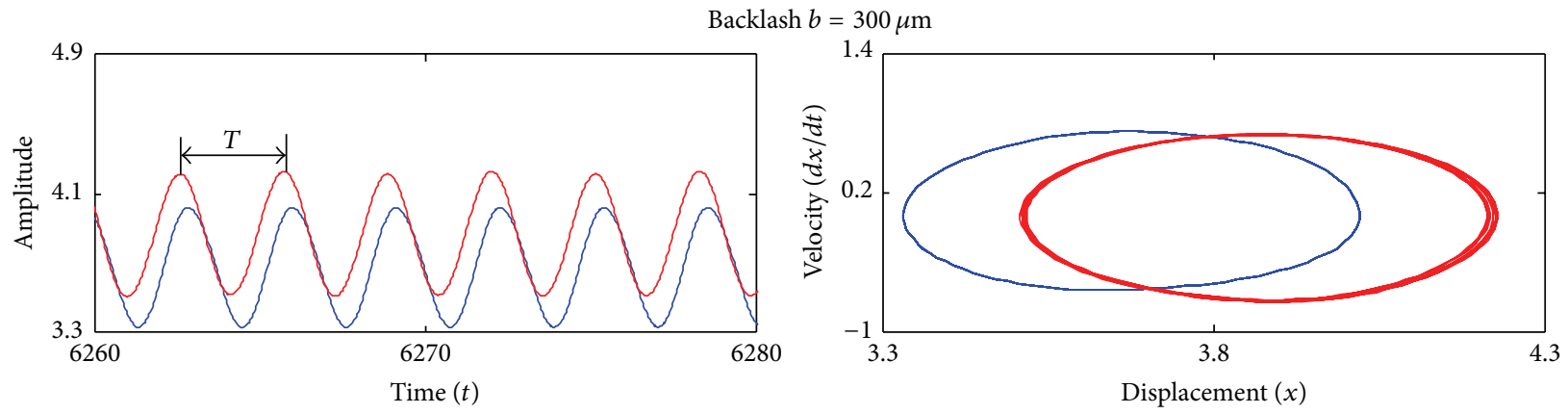

- Microsegment

- Involute

(a) Time history chart

— Microsegment

Involute

(b) Phase diagram

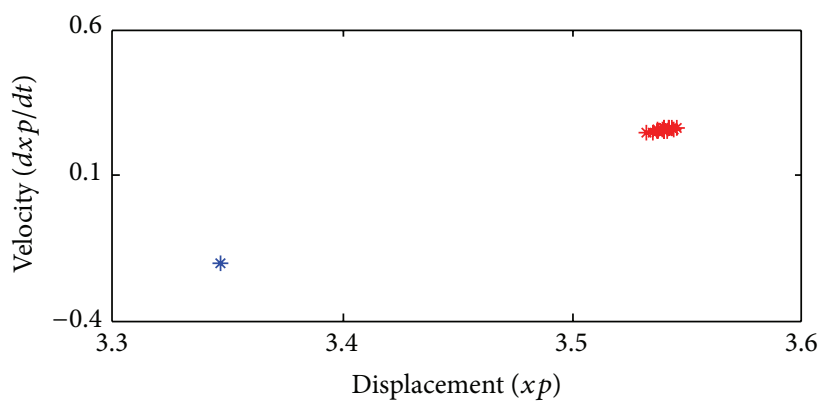

* Microsegment

* Involute

(c) Poincare perception mapping

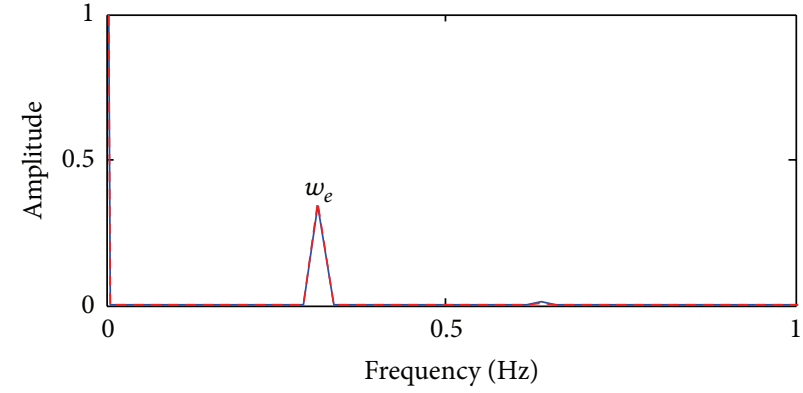

— Microsegment

- - - Involute

FIgURE 9: Dynamic performance comparison at $\Omega=2, T=1000 \mathrm{~N} \cdot \mathrm{m}$.
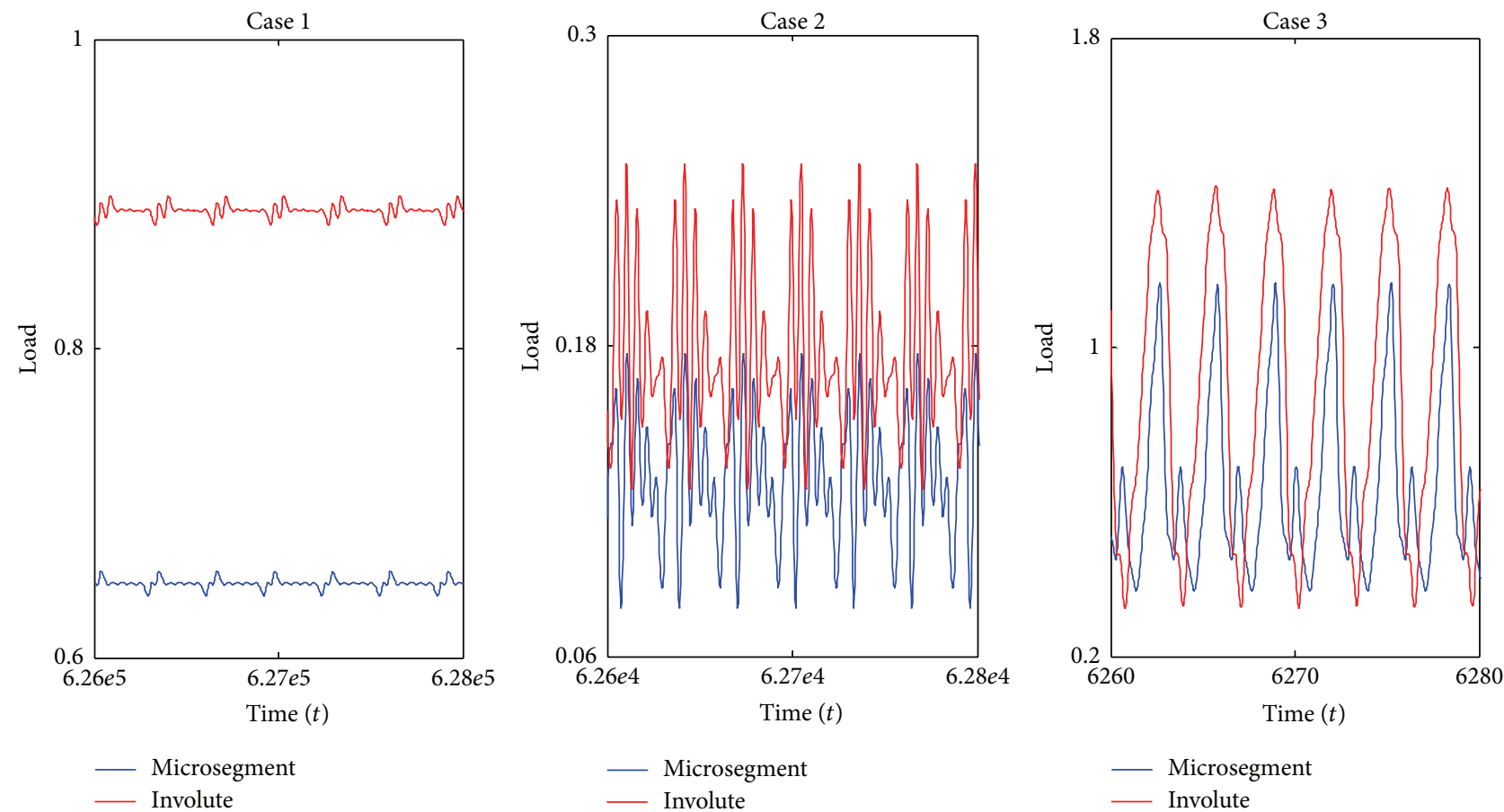

FIGURE 10: Dimensionless dynamic meshing loads for three cases. 


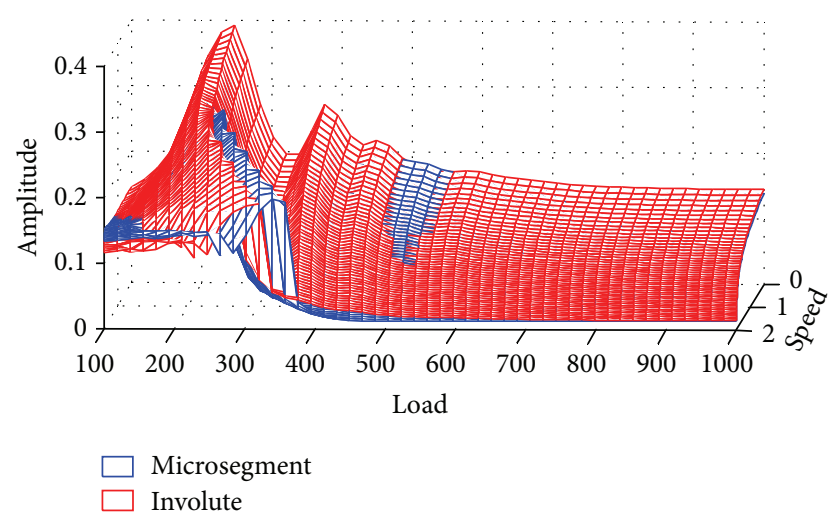

FIGURE 11: RMS of the vibration amplitude.

In general, the microsegment gear has the advantages in reducing the vibration amplitude and dynamic coefficient, and in HSHL condition, the periodicity and stability of microsegment gear are better than those of involute gear.

\section{Conclusions}

This paper introduced the new type microsegment gear transmission. Its principle and tooth profile equation are expounded. The dynamic model for microsegment gear transmission is developed by regarding the profile deviation as displacement excitation and the nonlinear dynamic analysis is carried out. The conclusions of this study are as follows:

(1) It is concave-convex contact in the meshing process of microsegment gear, and it is obvious that the root of microsegment gear is wider than that of involute gear. The mesh stiffness calculated through FEM is better than that of the involute gear.

(2) Due to the special profile, the traditional dynamic model of involute gear is unsuitable to be applied directly on microsegment gear. The proposed dynamic model for microsegment gear treats the profile deviation as displacement excitation. This is expected to establish an available method for the dynamic analysis of most of the noninvolute gear transmission.

(3) The nonlinear dynamics for both microsegment gear and involute gear are discussed. The numerical analysis results show that microsegment and involute gear systems have the same response, that is, in LSHL and MSML condition; the systems are in a nonharmonic single periodic motion both with strong periodicity and stability; in HSHL condition, the systems come into a simple harmonic motion, and the periodicity and stability for microsegment gear are better than these of involute gear according to the degree of concentration of the points in Poincare map. The comparison results on dynamic coefficient and RMS of the amplitude put in evidence the advantages of using microsegments gear for most of the working conditions.
(4) The influence of backlash on the dynamic performance is studied. In general, the variation of backlash does not change the type of motion, as well as the periodicity and stability of the motions in LSHL and MSML condition, but the vibration range moves up/down as the backlash increases/decreases; in HSHL condition, the dynamic performance of microsegment gear gets worse first and then gets worse as the backlash increases from $100 \mu \mathrm{m}$ to $300 \mu \mathrm{m}$.

(5) Insufficient research limits the development and application of microsegment gear. Hobbing is the most important approach for the processing of microsegment gear at the present. The amount of segments results in a great trouble on gear grinding, while the main idea of this paper presents a new technique of grinding with the profile deviation as the machining allowance.

\section{Conflict of Interests}

The authors declare that there is no conflict of interests regarding the publication of this paper.

\section{Acknowledgments}

This work is supported by International S\&T Cooperation Program of China (2014DFA80440) and Natural Science Foundation of Anhui Province of China (no. 1408085MKL12).

\section{References}

[1] S. P. Radzevich and D. W. Dudley, Handbook of Practical Gear Design, CRC Press, 1994.

[2] O. Alipiev, "Geometric design of involute spur gear drives with symmetric and asymmetric teeth using the Realized Potential Method," Mechanism and Machine Theory, vol. 46, no. 1, pp. 1032, 2011.

[3] G. Hyatt, M. Piber, N. Chaphalkar et al., "A review of new strategies for gear production," Procedia CIRP, vol. 14, pp. 7276, 2014.

[4] Y. Fujisawa and M. Komori, "Surface finishing method for tooth flank of heat-treated surface-hardened small gears using a gearshaped tool composed of alumina-fiber-reinforced plastic," Precision Engineering, vol. 39, pp. 234-242, 2015.

[5] R. Och, "History of gear measurement-measuring machines and traceability 1900-2005," Gear Product News, 2006.

[6] N. Ferreira, T. Krah, D. C. Jeong et al., "Integration of a silicon-based microprobe into a gear measuring instrument for accurate measurement of micro gears," Measurement Science and Technology, vol. 25, no. 6, Article ID 064016, 2014.

[7] ISO Standard, "Cylindrical gears-ISO system of accuracypart 1: definitions and allowable values of deviations relevant to corresponding flanks of gear teeth," ISO 1328-1, 1997.

[8] R. Larsson, "Transient non-Newtonian elastohydrodynamic lubrication analysis of an involute spur gear," Wear, vol. 207, no. 1-2, pp. 67-73, 1997. 
[9] Q. Y. Jiang and G. C. Barber, "Modeling of reaction film failure in gear lubrication," Wear, vol. 231, no. 1, pp. 71-76, 1999.

[10] B. R. Höhn, K. Michaelis, and H. P. Otto, "Minimised gear lubrication by a minimum oil/air flow rate," in Proceedings of the ASME International Design Engineering Technical Conferences and Computers and Information in Engineering Conference, pp. 717-725, American Society of Mechanical Engineers, 2007.

[11] H. Czichos, Tribology: A Systems Approach to the Science and Technology of Friction, Lubrication, and Wear, Elsevier, 2009.

[12] A. Kahraman and R. Singh, "Non-linear dynamics of a spur gear pair," Journal of Sound and Vibration, vol. 142, no. 1, pp. 49-75, 1990.

[13] R. G. Parker, S. M. Vijayakar, and T. Imajo, "Non-linear dynamic response of a spur gear pair: modelling and experimental comparisons," Journal of Sound and Vibration, vol. 237, no. 3, pp. $435-455,2000$.

[14] S. Theodossiades and S. Natsiavas, "Non-linear dynamics of gear-pair systems with periodic stiffness and backlash," Journal of Sound and Vibration, vol. 229, no. 2, pp. 287-310, 2000.

[15] M. Vaishya and R. Singh, "Analysis of periodically varying gear mesh systems with Coulomb friction using Floquet theory," Journal of Sound and Vibration, vol. 243, no. 3, pp. 525-545, 2001.

[16] V. K. Tamminana, A. Kahraman, and S. Vijayakar, "A study of the relationship between the dynamic factors and the dynamic transmission error of spur gear pairs," Journal of Mechanical Design, vol. 129, no. 1, pp. 75-84, 2007.

[17] T. Osman and P. Velex, "A model for the simulation of the interactions between dynamic tooth loads and contact fatigue in spur gears," Tribology International, vol. 46, no. 1, pp. 84-96, 2012.

[18] T. Komori, R. Ariga, and S. Nagata, "A new profile of relative curvature being zero at many contact points," in Proceedings of the International Conference on Gearing, vol. 1, pp. 39-42, CMES, Beijing, China, 1988.

[19] T. Komori, R. Ariga, and S. Nagata, "A new gear profile having zero relative curvature at many contact points (Logix tooth profile)," in Proceedings of the International Power Transmission and Gearing Conference, vol. 2, pp. 599-606, ASME, Chicago, Ill, USA, April 1989.

[20] Z. Han, L. Jinhua, L. Hongyu, and C. Anshi, "Constructing principle and features of tooth profiles with micro-segment," Chinese Journal of Mechanical Engineering, vol. 33, pp. 7-11, 1997.

[21] K. Huang, Theoretical and experimental research of microsegment gear [Ph.D. thesis], Hefei University of Technology, Hefei, China, 2002.

[22] K. Huang, H. Zhao, and J. Tian, "Experimental research on temperature rise comparison between micro-segment gear and involute gear," China Mechanical Engineering, vol. 17, no. 18, pp. 1880-1883, 2006.

[23] Q. Chen, H. Zhao, and K. Huang, "Study on calculation of transmission efficiency about the micro-segment gear," China Mechanical Engineering, vol. 22, no. 13, pp. 1537-1539, 2011.

[24] M. Blagojević, V. Nikolic, N. Marjanović et al., "Analysis of cycloid drive dynamic behavior," Scientific Technical Review, vol. 59, no. 1, pp. 52-56, 2009.

[25] H. N. Özgüven and D. R. Houser, "Mathematical models used in gear dynamics-a review," Journal of Sound and Vibration, vol. 121, no. 3, pp. 383-411, 1988.

[26] A. Farshidianfar and A. Saghafi, "Global bifurcation and chaos analysis in nonlinear vibration of spur gear systems," Nonlinear Dynamics, vol. 75, no. 4, pp. 783-806, 2014.
[27] O. D. Mohammed, M. Rantatalo, and J.-O. Aidanpää, "Dynamic modelling of a one-stage spur gear system and vibration-based tooth crack detection analysis," Mechanical Systems and Signal Processing, vol. 54, pp. 293-305, 2015.

[28] M. Inalpolat, M. Handschuh, and A. Kahraman, "Influence of indexing errors on dynamic response of spur gear pairs," Mechanical Systems and Signal Processing, vol. 60-61, pp. 391405, 2015. 


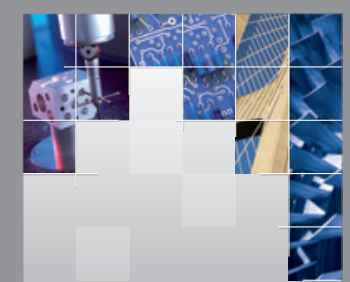

\section{Enfincering}
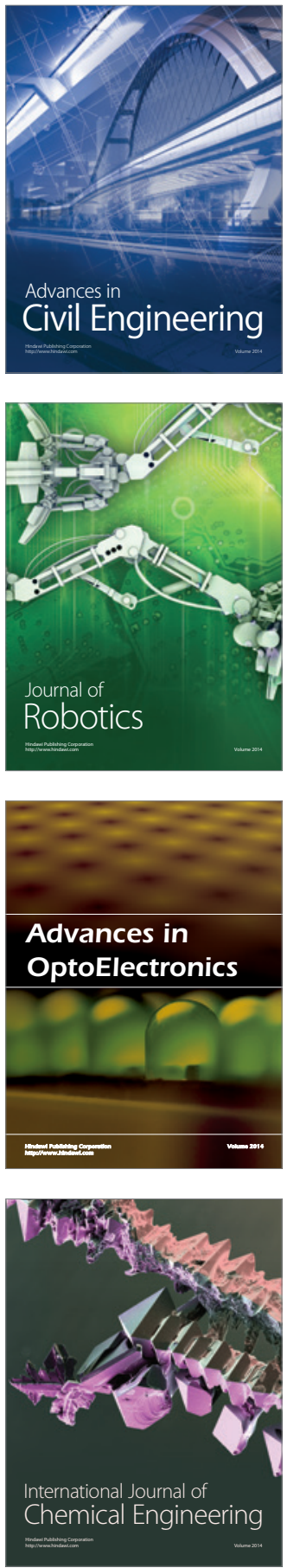

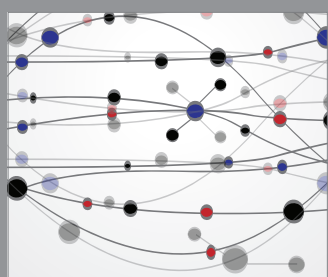

The Scientific World Journal

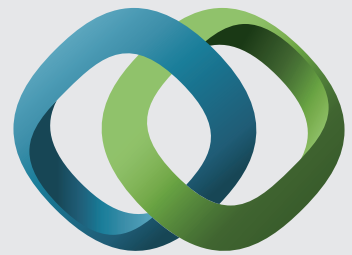

\section{Hindawi}

Submit your manuscripts at

http://www.hindawi.com
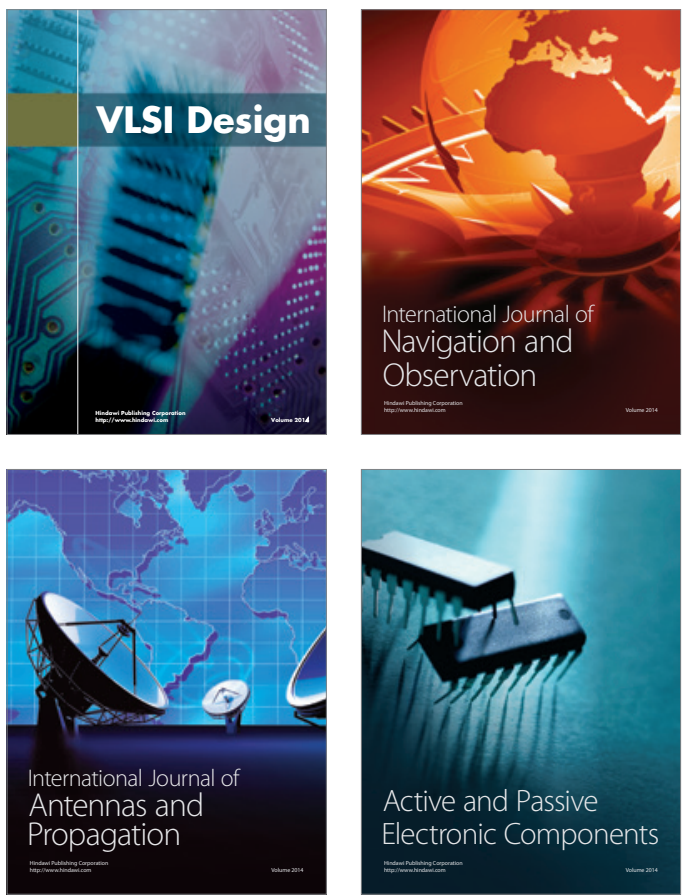
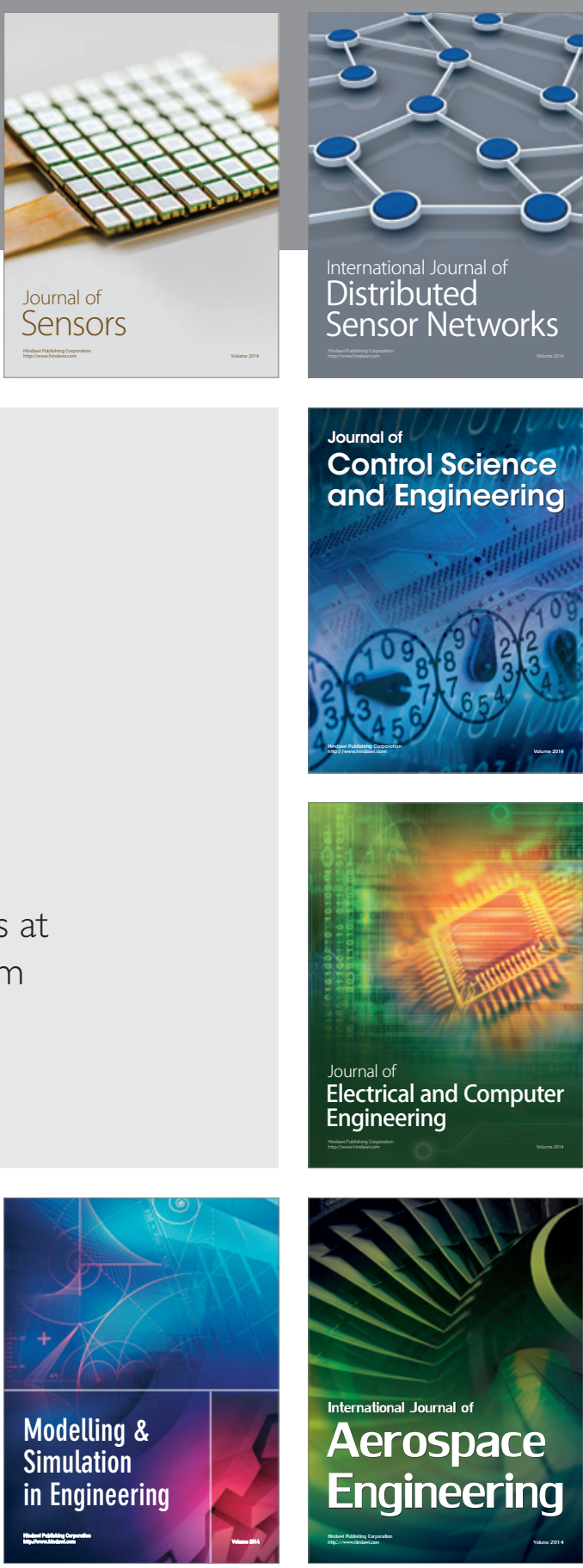

International Journal of

Distributed

Sensor Networks

Journal of

Control Science

and Engineering
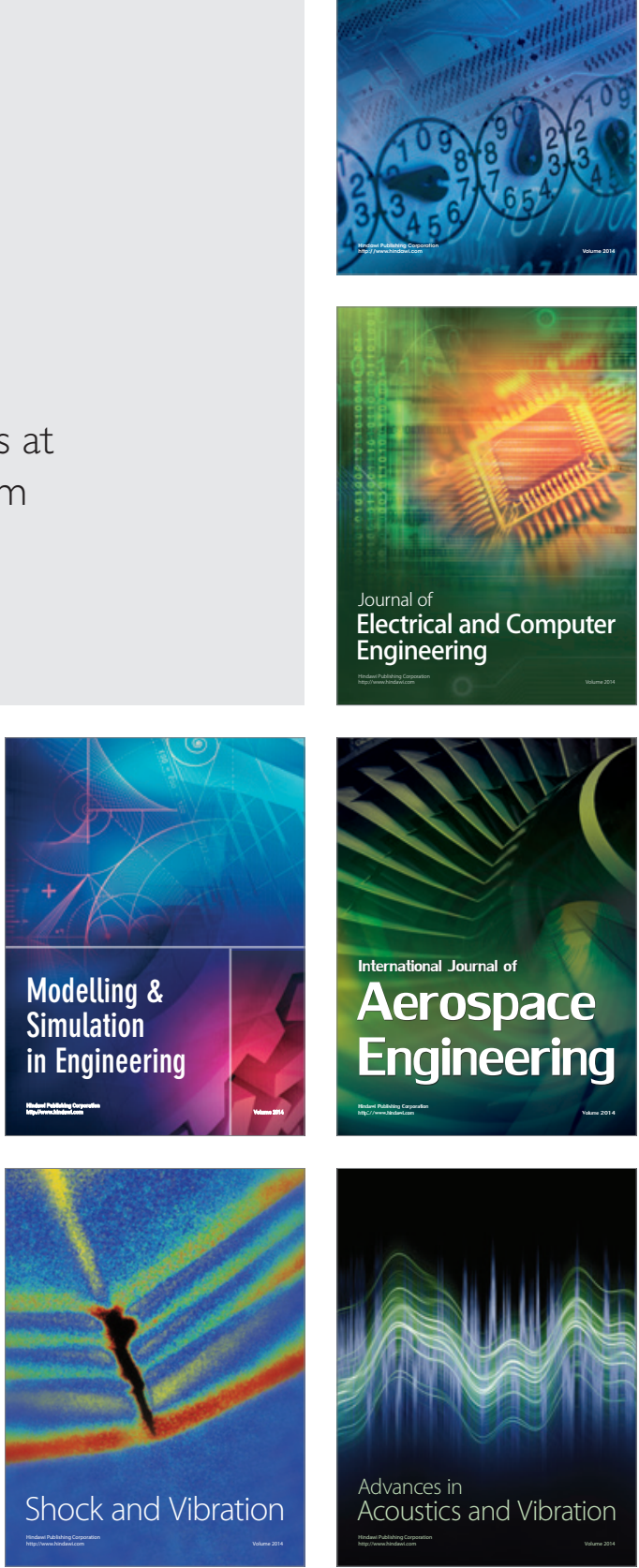